\title{
Understanding Quantum Entanglement: Qubits, Rebits and the Quaternionic Approach ${ }^{1}$
}

\author{
J. Batle*, , A. R. Plastino*,**,***, M. Casas*, and A. Plastino**,*** \\ * Departament de Física, Universitat de les Illes Balears, 07071 Palma de Mallorca, Spain \\ ** Facultad de Astronomía y Geofísica, Universidad Nacional de La Plata and CONICET, \\ C.C. 727, 1900 La Plata, Argentina \\ *** Departamento de Física, Universidad Nacional de la Plata and CONICET, \\ C.C. 727, 1900 La Plata, Argentina \\ e-mail:vdfsjbv4@uib.es. \\ Received November 25, 2002
}

\begin{abstract}
It has been recently pointed out by Caves, Fuchs, and Rungta [1] that real quantum mechanics (that is, quantum mechanics defined over real vector spaces [2-5]) provides an interesting foil theory whose study may shed some light on just which particular aspects of quantum entanglement are unique to standard quantum theory and which are more generic over other physical theories endowed with this phenomenon. Following this work, some entanglement properties of two-rebit systems are discussed and a comparison with the basic properties of two-qubit systems, i.e., the systems described by standard complex quantum mechanics, is made. The use of quaternionic quantum mechanics as applied to the phenomenon of entanglement is also discussed. (C) 2003 MAIK "Nauka/Interperiodica".
\end{abstract}

\section{INTRODUCTION}

The phenomenon of entanglement is one of the most characteristic nonclassical features exhibited by quantum systems [6]. Quantum entanglement is the basic source of several quantum processes, such as superdense coding [7], quantum teleportation [8] and quantum computation $[6,9]$, studied by quantum information theory [10-13]. A state of a composite quantum system constituted by subsystems $A$ and $B$ is called "entangled" if it cannot be represented as a mixture of factorizable pure states.

The simplest systems exhibiting the phenomenon of entanglement are two-qubit systems. For these systems, an explicit expression of the entanglement of formation, which is a natural quantitative measure of entanglement [14], has been found by Wootters [15]. The corresponding space of mixed two-qubit states in standard quantum mechanics is 15-dimensional. The amount of entanglement in this space has been established in [16-19].

For quantum mechanics defined over real vector spaces, the simplest composite systems are two-rebit systems. An explicit expression for the entanglement of formation of arbitrary states of two rebits has been obtained by Caves, Fuchs, and Rungta [1]. Pure states of rebit systems are described by normalized vectors in a two-dimensional real vector space. The correspond-

\footnotetext{
1 This article was submitted by the authors in English

${ }^{2}$ Corresponding author.
}

ing space of mixed two-rebit states is 9-dimensional (versus 15 for two-qubit states).

The aim of the present work is to explore numerically and conceptually the entanglement properties of two-rebit systems [20] as compared to the usual twoqubit states, so as to detect the differences between the two types of systems. We pay particular attention to the distribution of states according to their degree of entanglement. We obtain, analytically, the probability densities for finding pure states with a given amount of entanglement $E$ (or with fixed values of the so-called concurrence squared $C^{2}$ ). The same is done for mixed states in numerical fashion.

The paper is organized as follows. In Section 2, we review the general properties of two-qubit systems. Several quantities related to the entanglement of formation are investigated for both rebits and qubits in Section 3, with an emphasis on the differences and similarities between both formalisms. In section 4, we discuss how to follow a quaternionic approach to quantum entanglement. Finally, some conclusions are drawn in Section 5 .

\section{ENTANGLEMENT OF TWO-QUBIT SYSTEMS}

As already stressed in the Introduction, the twoqubit system is the simplest quantum mechanical system that exhibits between the two parties the sort of "extra correlation" referred to as entanglement. A representation of a qubit is given by the Bloch sphere. The poles correspond to the classical bits $|0\rangle,|1\rangle$, while any 
point on this unit sphere, given by a pair of angles $\phi, \psi$, represents a general qubit $\cos \phi|0\rangle+e^{i \psi} \sin \phi|1\rangle$. Qubits constitute essential new elements in the switch from classical to quantum information theory and entanglement the basic ingredient of many striking processes now under active investigation.

Here, we use the concurrence $C[\hat{\rho}]$ and the entanglement of formation $E[\hat{\rho}]$ as quantitative measures of the amount of entanglement. Wootters' formula for the entanglement of formation of a two-qubit state $\hat{\rho}$ reads [15]

$$
E[\hat{\rho}]=h\left(\frac{1+\sqrt{1-C^{2}}}{2}\right),
$$

where

$$
h(x)=-x \log _{2} x-(1-x) \log _{2}(1-x),
$$

and the concurrence $C$ is given by $C=\max \left(0, \lambda_{1}-\lambda_{2}-\right.$ $\left.\lambda_{3}-\lambda_{4}\right)$, with $\lambda_{i}(i=1, \ldots, 4)$ being the square roots, in decreasing order, of the eigenvalues of the matrix $\hat{\rho} \tilde{\rho}$, with

$$
\tilde{\rho}=\left(\sigma_{y} \otimes \sigma_{y}\right) \rho^{*}\left(\sigma_{y} \otimes \sigma_{y}\right) .
$$

The above expression has to be evaluated by recourse to the matrix elements of $\hat{\rho}$, computed with respect to the conventional product basis.

In this work, we have generated all pure and mixed states of a two-qubit system according to the measure defined in $[16,17,21]$.

The distribution of two-qubit states according to their degree of mixture can be obtained analytically by adopting a geometric representation [22] for the eigenvalues of $\hat{\rho}$ as a regular tetrahedron $T_{\Delta}$ of side length 1 , in $\mathscr{R}^{3}$, centered at the origin. There exists a mapping connecting these eigenvalues $\left(p_{1}, \ldots, p_{4}\right)$ with the points $\mathbf{r}$ of the tetrahedron, so that we can relate the participation ratio $R(\hat{\rho})=1 / \operatorname{Tr}\left(\hat{\rho}^{2}\right)$ to the radius $r=|\mathbf{r}|$ of a sphere embedded within the tetrahedron $T_{\Delta}$ according to

$$
r^{2}=-\frac{1}{8}+\frac{1}{2} \sum_{i=1}^{4} p_{i}^{2} .
$$

Thus, the states with a given degree of mixture lie on the surface of a sphere of radius $r$ concentric with the tetrahedron $T_{\Delta}$ (for a complete description, see [22]). It is then easy to find out just how our states are so distributed, according to the degree of mixture $R$. This distribution is depicted in Fig. 1. The dualistic nature of the concepts of entanglement and mixedness becomes apparent. For two-qubit systems, one would expect that the states will tend to become less entangled as the degree of mixture increases. In fact, for $R \geq 3$ (vertical line in Fig. 1), all states are separable. It is important to

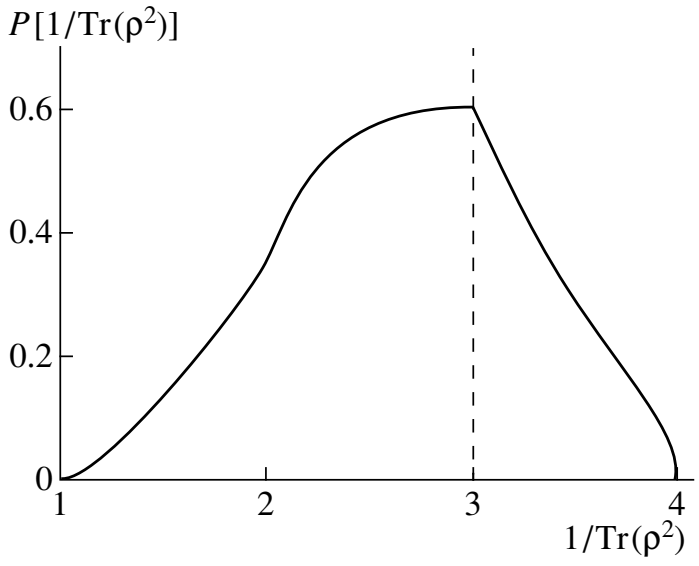

Fig. 1. Probability (density) distribution for finding a state $\hat{\rho}$ with a given participation ratio $R$. States (two-qubit) with $R \geq 3$ are always separable.

stress here that this curve is the same no matter what quantities (rebits, qubits, or even quaterbits) are involved, although their respective entanglement properties are different.

\section{ENTANGLEMENT PROPERTIES OF TWO-REBIT SYSTEMS: A COMPARISON}

In the space of real quantum mechanics, we can represent rebits on the Bloch sphere. The poles correspond to the classical bits $|0\rangle,|1\rangle$, but the sphere reduces itself now to a maximum unit circle, described by just one parameter $\phi$. We have $\cos \phi|0\rangle+e^{i \psi} \sin \phi|1\rangle \longrightarrow \cos \phi|0\rangle+$ $\sin \phi|1\rangle$. Entanglement can also be described in such a context with suitable modifications. The Caves-FuchsRungta (CFR) formula for the entanglement of formation of a two-rebit state $\rho$ is given by (1), (2) with the concurrence $C[\rho]=|\operatorname{Tr}(\tau)|=\left|\operatorname{Tr}\left(\rho \sigma_{y} \otimes \sigma_{y}\right)\right|$, which has to be evaluated using the matrix elements of $\rho$ computed with respect to the product basis, $|i, j\rangle=|i\rangle|j\rangle, i$, $j=0,1$.

For a two-rebit state, the entanglement of formation is completely determined by the expectation value of one single observable, namely, $\sigma_{y} \otimes \sigma_{y}$.n the contrary, it has recently been proved that there is no observable (not even for pure states) whose sole expectation value will yield enough information to determine the entanglement of a two-qubit state [23]. As shown in [20], there are mixed states of two rebits with maximum entanglement (that is, with $C^{2}=1$ ) within the range $1 \leq$ $R \leq 2$. This is clearly in contrast to what happens with two-qubit states, because only pure states $(R=1)$ have maximum entanglement.

The measure of mixedness $R$ introduced above can be used in the description of the entanglement phenomenon for two-rebit systems. Also, in order to explore numerically the properties of arbitrary two-rebit states, it is necessary to introduce an appropriate measure $\mu$ on 

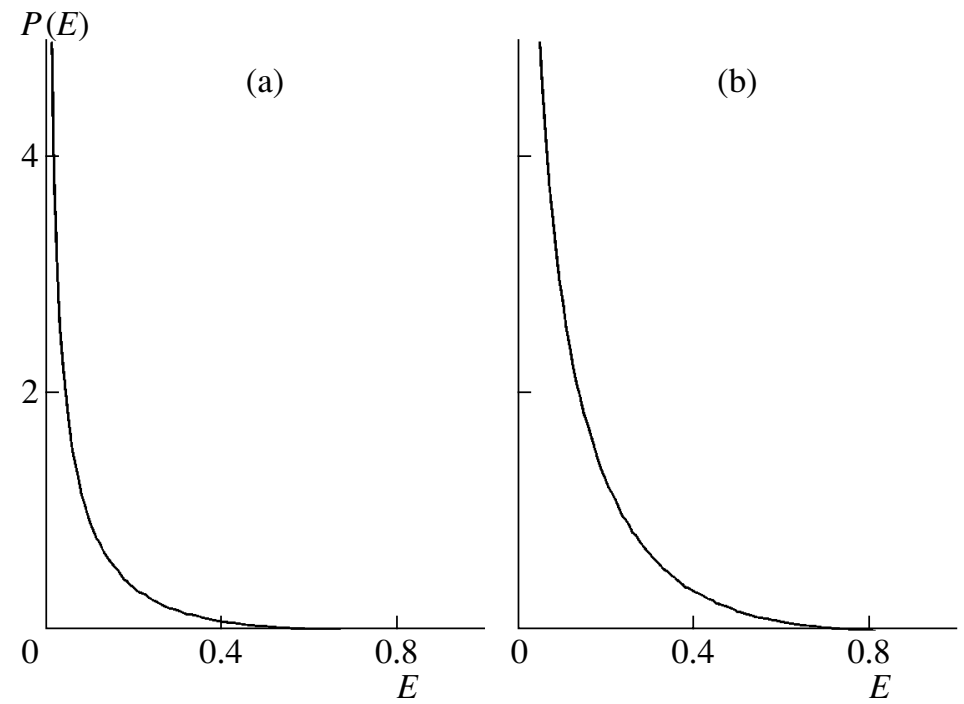

Fig. 2. Probability (density) distributions for finding a state $\hat{\rho}$ with a given amount of entanglement $E$ : (a) for qubits; (b) for rebits.

the space $\mathscr{S}_{R}$ of general two-rebit states. Such a measure is needed to compute volumes within the space $\mathscr{Y}_{R}$, as well as to determine what is to be understood by a uniform distribution of states on $\mathscr{Y}_{R}[16,17]$.

An arbitrary (pure and mixed) state $\rho$ of a (real) quantum system described by an $N$-dimensional real Hilbert space can always be expressed as the product of three matrices,

$$
\rho=R D\left[\left\{\lambda_{i}\right\}\right] R^{T}
$$

Here, $R$ is an $N \times N$ orthogonal matrix and $D\left[\left\{\lambda_{i}\right\}\right]$ is an $N \times N$ diagonal matrix whose diagonal elements are $\left\{\lambda_{1}, \ldots, \lambda_{N}\right\}$, with $0 \leq \lambda_{i} \leq 1$, and $\sum_{i} \lambda_{i}=1$. The group of orthogonal matrices $O(N)$ is endowed with a unique, uniform measure $v$ [21]. At the same time, the simplex $\Delta$, consisting of all the real $N$-uples $\left\{\lambda_{1}, \ldots, \lambda_{N}\right\}$ appearing in (5), is a subset of an $(N-1)$-dimensional hyperplane of $\mathscr{R}^{N}$. Consequently, the standard normalized Lebesgue measure $\mathscr{L}_{N-1}$ on $\mathscr{R}^{N-1}$ provides a natural measure for $\Delta$. The aforementioned measures on $O(N)$ and $\Delta$ lead then to a natural measure $\mu=v \mathscr{L}_{N-1}$ on the set $\mathscr{Y}_{R}$ of all the states of our (real) quantum system.

Clearly, our system will have $N=4$ again, and in our numerical computations we are going to randomly generate states of a two-rebit system according to the measure $\mu$.

The relationship between the amount of entanglement and the purity of quantum states of composite systems has recently been discussed in the literature [1619]. The amount of entanglement and the purity of quantum states of composite systems exhibit a dualistic relationship. As the degree of mixture increases, quantum states tend to have a smaller amount of entanglement. In the case of two-qubit systems, states with a large enough degree of mixture are always separable [16]. To study the relationship between entanglement and mixture in real quantum mechanics, we compute numerically the probability $P(E)$ of finding a two-rebit state endowed with an amount of entanglement $E$. In Fig. 2, we compare (i) the distribution associated with two-rebit states with (ii) that associated with two-qubit states recently obtained by Zyczkowski et al. [16, 24]. Figure $2 \mathrm{a}$ depicts the probability $P(E)$ of finding twoqubit states endowed with a given entanglement $E$ (as computed with Wootters' expression). In a similar way, Fig. 2b exhibits a plot of the probability $P(E)$ of finding two-rebit states endowed with a given entanglement $E$ (as computed with the CFR formula). Comparing Figs. $2 \mathrm{a}$ and $2 \mathrm{~b}$, we find that the distributions $P(E)$ describing arbitrary states (that is, both pure and mixed states) exhibit the same qualitative shape for both twoqubit and two-rebit states: in both cases, the distribution $P(E)$ is a decreasing function of $E$.

The distribution $P(E)$ or $P\left(C^{2}\right)$ for pure two-rebit states can be obtained analytically. Let us write a pure two-rebit state in the form

$$
|\Psi\rangle=\sum_{i=1}^{4} c_{i}\left|\phi_{i}\right\rangle,
$$

where

$$
\sum_{i=1}^{4} c_{i}^{2}=1, \quad c_{i} \in \mathscr{R} .
$$

The states $\left(\left|\phi_{i}\right\rangle, i=1, \ldots, 4\right)$ are the eigenstates of the operator $\sigma_{y} \otimes \sigma_{y}$. The four real numbers $c_{i}$ constitute the coordinates of a point lying on the three-dimensional unitary hypersphere $S_{3}$ (which is embedded in 


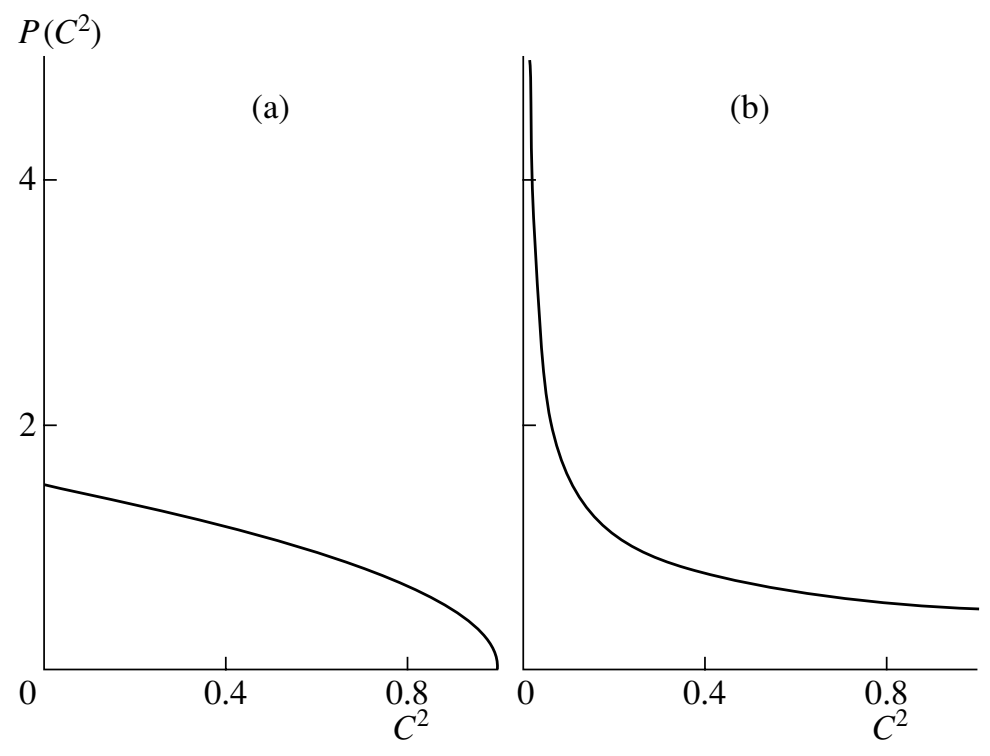

Fig. 3. Probability distributions for finding a state $\hat{\rho}$ with a given concurrence squared $C^{2}$ : (a) for qubits; (b) for rebits.

$\mathscr{R}^{4}$ ). We now introduce on $S_{3}$ three angular coordinates, $\phi_{1}, \phi_{2}$, and $\theta$, defined by

$$
\begin{aligned}
& c_{1}=\cos \theta \cos \phi_{1}, \\
& c_{2}=\cos \theta \sin \phi_{1}, \\
& c_{3}=\sin \theta \cos \phi_{2}, \\
& c_{4}=\sin \theta \sin \phi_{2}, \\
& 0 \leq \theta<\pi / 2, \quad 0 \leq \phi_{1}, \quad \phi_{2}<2 \pi .
\end{aligned}
$$

In terms of the above angular coordinates, the concurrence of the pure state $|\Psi\rangle$ is given by

$$
C=\left|\left\langle\sigma_{y} \otimes \sigma_{y}\right\rangle\right|=|\cos 2 \theta| .
$$

Using (8) and (9), one deduces that the probability density $P\left(C^{2}\right)$ of finding a pure two-rebit state with a squared concurrence $C^{2}$ is given by

$$
P\left(C^{2}\right)=\frac{1}{2 \sqrt{C^{2}}}
$$

The distribution is to be compared with the one obtained for pure states of two-qubit systems, which is (analytically) found to be [24]

$$
P\left(C^{2}\right)=\frac{3}{2} \sqrt{1-C^{2}} .
$$

Both distributions are compared in Fig. 3. Figure 3a depicts the one for qubits, while Fig. $3 b$ shows the distribution for rebits. The distribution remains finite, in the case of qubits, for all $C^{2}$. In the case of rebits, it presents a sharp peak at the origin and then levels off at $1 / 2$ at $C^{2}=1$. The general conclusion that one can draw from Figs. 2 and 3 is that the curves representing the distributions $P(E)$ and $P\left(C^{2}\right)$ associated with (i) pure states and (ii) arbitrary states do not differ, in the case of two-rebit states, as much as they do in the case of two-qubit states.

We can determine analytically which is the maximum entanglement $E_{m}$ of a two-rebit state compatible with a given participation ratio $R$. Since $E$ is a monotonic increasing function of the concurrence $C$, we shall find the maximum value of $C$ compatible with a given value of $R$. In order to solve the ensuing variational problem (and bearing in mind that $C=\left|\left\langle\sigma_{y} \otimes \sigma_{y}\right\rangle\right|$ ), let us first find the state that extremizes $\operatorname{Tr}\left(\rho^{2}\right)$ under the constraints associated with a given value of $\left\langle\sigma_{y} \otimes \sigma_{y}\right\rangle$ and the normalization of $\rho$. This variational problem can be cast in the fashion

$$
\delta\left[\operatorname{Tr}\left(\rho^{2}\right)+\beta\left\langle\sigma_{y} \otimes \sigma_{y}\right\rangle-\alpha \operatorname{Tr}(\rho)\right]=0,
$$

where $\alpha$ and $\beta$ are appropriate Lagrange multipliers.

After some algebra and expressing the expectation value of $\left\langle\sigma_{y} \otimes \sigma_{y}\right\rangle$ in terms of the parameter $\beta$, one finds that the maximum value of $C^{2}$ compatible with a given value of $R$ is given by

$$
C_{m}^{2}=\left\{\begin{array}{l}
1, \quad 1 \leq R \leq 2 \\
4 / R-1, \quad 2 \leq R \leq 4 .
\end{array}\right.
$$

\section{THE QUATERNIONIC APPROACH TO QUANTUM ENTANGLEMENT}

The quaternionic space $\mathscr{H}$ constitutes a generalization of the complex space $\mathscr{C}$, which, in turn, generalizes the real space $\mathscr{R}$. Each step of this chain is made possible by introducing new quantities: $i^{2}=-1$ from $\mathscr{R}$ to $\mathscr{C}$ 



Fig. 4. Plot of the entanglement of pure states in the framework of real quantum mechanics (RQM), standard quantum mechanics (SQM), and quaternionic quantum mechanics (QQM). See text for details. $x=C_{10}^{2}+C_{11}^{2} ; y=C_{12}^{2}+C_{13}^{2} ; N^{2}\left(C_{1}\right)=C_{10}^{2}+C_{11}^{2}+$ $C_{12}^{2}+C_{13}^{2}$.

and $j^{2}=k^{2}=-1$ from $\mathscr{C}$ to $\mathscr{H}$, with suitable commutation laws for the three quantities $i, j, k$.

A general quaternion $\phi$ and its associated commutation algebra are written in the following fashion:

$$
\phi=\phi_{0}+i \phi_{1}+j \phi_{2}+k \phi_{3},
$$

with $\phi_{i} \in \mathscr{R}$ and $i j=-j i=k, j k=-k j=i, k i=-i k=j$.

This "natural extension" of complex numbers that yields quaternions cannot be generalized any further. Thus, if we give up the property of commutativity, the most general algebra that can be used in quantum mechanics is the quaternionic one [25].

\section{Entanglement for Pure States of Two- "Quaterbit" Systems}

The definition of entanglement in quaternionic quantum mechanics (QQM) for pure states does not differ from the standard one. Given a pure state $|\psi\rangle$ of a composite bipartite system, the entanglement is obtained via the von Neumann entropy of the marginal density matrix associated with the subsystem $A$ by tracing over the subsystem $B: \hat{\rho}_{A}=\operatorname{Tr}_{B}|\psi\rangle_{A B}\langle\psi|$ of the total density matrix $\hat{\rho}=|\psi\rangle_{A B}\langle\psi|$ or, vice versa, $\hat{\rho}_{B}=$ $\operatorname{Tr}_{A}|\psi\rangle_{A B}\langle\psi|$. Thus, $E(\hat{\rho})=S\left(\hat{\rho}_{A}\right)=S\left(\hat{\rho}_{B}\right)$.

In the case of quaternions, we face a higher dimensionality and, therefore, we need more parameters to describe the state $\hat{\rho}$. Additionally, in using the kets and bras notation of Dirac, we must keep in mind the non- commutativity rules of quaternions. For the sake of simplicity, let us suppose that a pure state is written as

$$
\begin{gathered}
|\psi\rangle=C_{1}|0\rangle_{A}|0\rangle_{B}+C_{2}|1\rangle_{A}|1\rangle_{B} \\
C_{1}, C_{2} \in \mathcal{H}, \quad\left|C_{1}\right|^{2}+\left|C_{2}\right|^{2}=1 .
\end{gathered}
$$

The statistical matrix $\hat{\rho}=|\psi\rangle_{A B}\langle\psi|$ reads

$$
\left(\begin{array}{c}
C_{1} \\
0 \\
0 \\
C_{2}
\end{array}\right)\left(\begin{array}{llll}
\overline{C_{1}} & 0 & 0 & \overline{C_{2}}
\end{array}\right)=\left(\begin{array}{cccc}
C_{1} \overline{C_{1}} & 0 & 0 & C_{1} \overline{C_{2}} \\
0 & 0 & 0 & 0 \\
0 & 0 & 0 & 0 \\
C_{2} \overline{C_{1}} & 0 & 0 & C_{2} \overline{C_{2}}
\end{array}\right),
$$

with $\hat{\rho}^{\dagger}=\hat{\rho}$ since $\overline{C_{1} \overline{C_{2}}}=\overline{\overline{C_{2}}} \overline{C_{1}}=C_{2} \overline{C_{1}}$. Entanglement then is only a function of $N\left(C_{1}\right)^{2}=C_{10}^{2}+C_{11}^{2}+C_{12}^{2}+C_{13}^{2}$. Identifying $x \equiv C_{10}^{2}+C_{11}^{2}$ and $y \equiv C_{12}^{2}+C_{13}^{2}$, we plot $E(\hat{\rho})$ in Fig. 4, together with $E(\hat{\rho})$ for the three versions of quantum mechanics we are dealing with here.

\section{Extension to General Mixed States}

The full analytical study of entanglement in the framework of quaternionic quantum mechanics requires careful consideration of the algebra of states and operators for these "hypernumbers." If one wishes to discuss how to carry out statistical studies and how entanglement-related properties are distributed over the 
space of all (pure and mixed) states, one notices that, in this case, the dimensionality of the problem for a general $4 \times 4$ matrix is substantially higher $(3+4 \times 6=27)$ than for the complex $(3+2 \times 6=15)$ or real $(3+1 \times$ $6=9$ ) cases. The ensuing statistical properties become clearly nontrivial, and some substantial effort is required.

Let us merely list here the basic ingredients needed for a complete description of the statistical properties of quaternionic states $\hat{\rho}$ : (i) building and correctly parametrizing the unitary transformations, defined over the quaternionic Hilbert space; (ii) specifying the form of the Haar measure for the concomitant space of unitary transformations. Notice that the measure on the simplex is exactly the same as for complex or real systems. For mixed states $\hat{\rho}$, the distributions associated with quantities that depend only on the eigenvalues of a statistical operator do have the same form in the real or the complex cases, for they depend only on the simplex. The results of the corresponding numerical study will be published elsewhere.

\section{CONCLUSIONS}

We have explored numerically the entanglement properties of two-rebit systems. A systematic comparison has been established between many statistical properties of two-qubit and two-rebit systems. We paid particular attention to the relationship between entanglement and purity in both quantum mechanical frameworks. We have also determined numerically the probability densities $P(E)$ of finding (i) pure two-rebit states and (ii) arbitrary two-rebit states endowed with a given amount of entanglement $E$ or concurrence squared $C^{2}$. In particular, we determined analytically the maximum possible value of the concurrence squared $C^{2}$ of two-rebit states compatible with a given value of mixedness $R$. As for the probability of finding states with a given amount of entanglement, the difference between mixed and pure states is much larger for qubits than for rebits. Also, we have sketched the manner in which quaternionic formalism could be applied to the study of quantum entanglement.

\section{ACKNOWLEDGMENTS}

This work was partially supported by the MCyT grant BFM2002-03241 (Spain) and by CONICET (Argentine Agency).

\section{REFERENCES}

1. C. M. Caves, C. A. Fuchs, and P. Rungta, Found. Phys. Lett. 14, 199 (2001).

2. E. C. G. Stueckelberg, Helv. Phys. Acta 33, 727 (1960).

3. M. Guenin, C. Piron, H. Ruegg, and E. C. G. Stueckelberg, Helv. Phys. Acta 34, 675 (1961).

4. G. G. Emch, Mathematical and Conceptual Foundations of 20th-Century Physics (North-Holland, Amsterdam, 1986).

5. W. K. Wootters, J. Math. Phys. 43, 4307 (2002).

6. Introduction to Quantum Computation and Information, Ed. by Hoi-Kwong Lo, S. Popescu, and T. Spiller (World Sci., River Edge, 1998).

7. C. H. Bennett and S. J. Wiesner, Phys. Rev. Lett. 69, 2881 (1992).

8. C. H. Bennett, G. Brassard, C. Crepeau, et al., Phys. Rev. Lett. 70, 1895 (1993).

9. A. Galindo and M. A. Martin-Delgado, Rev. Mod. Phys. 74, 347 (2002).

10. C. P. Williams and S. H. Clearwater, Explorations in Quantum Computing (Springer, New York, 1997).

11. Quantum Computing and Quantum Communications, Ed. by C. P. Williams (Springer, Berlin, 1998).

12. The Physics of Quantum Information, Ed. by D. Bouwmeester, A. Ekert, and A. Zeilinger (Springer, Berlin, 1998).

13. G. Alber, T. Beth, P. Horodecki, R. Horodecki, M. Röttler, H. Weinfurter, R. Werner, and A. Zeilinger, Quantum Information (Springer, Berlin, 2001), Springer Tracts Mod. Phys. 173.

14. C. H. Bennett, D. P. DiVicenzo, J. Smolin, and W. K. Wootters, Phys. Rev. A 54, 3824 (1996).

15. W. K. Wootters, Phys. Rev. Lett. 80, 2245 (1998).

16. K. Zyczkowski, P. Horodecki, A. Sanpera, and M. Lewenstein, Phys. Rev. A 58, 883 (1998).

17. K. Zyczkowski, Phys. Rev. A 60, 3496 (1999).

18. W. J. Munro, D. F. V. James, A. G. White, and P. G. Kwiat, Phys. Rev. A 64, 030302 (2001).

19. S. Ishizaka and T. Hiroshima, Phys. Rev. A 62, 022310 (2000).

20. J. Batle, M. Casas, A. R. Plastino, and A. Plastino, Phys. Lett. A 298, 301 (2002).

21. M. Pozniak, K. Zyczkowski, and M. Kus, J. Phys. A 31, 1059 (1998).

22. J. Batle, A. R. Plastino, M. Casas, and A. Plastino, Phys. Lett. A 296, 251 (2002).

23. J. M. G. Sancho and S. F. Huelga, Phys. Rev. A 61 , 042303 (2000).

24. K. Zyczkowski and H.-J. Sommers, J. Phys. A 34, 7111 (2001).

25. S. L. Adler, Quaternionic Quantum Mechanics and Quantum Fields (Oxford Univ. Press, New York, 1995). 